\title{
Effect of repeated locomotor training on ventilatory measures, perceived exertion and walking endurance in persons with motor incomplete spinal cord injury
}

\author{
Gino S. Panza $\mathbb{1}^{1,2} \cdot$ Andrew A. Guccione $\mathbb{1}^{3}$ \\ Received: 28 July 2020 / Revised: 22 September 2020 / Accepted: 23 September 2020 \\ (c) The Author(s), under exclusive licence to International Spinal Cord Society 2020
}

\begin{abstract}
Study design Pre-Post, Repeated Measures.

Objectives To determine if a warm-up bout of exercise could elicit a phasic ventilatory response to constant work rate (CWR) exercise in individuals with incomplete spinal cord injury (iSCI) during unsupported CWR treadmill walking. Describe the changes in ventilatory kinetics, ventilatory variability and ratings of perceived exertion (RPE) before and after 12 and 24 weeks of overground locomotor training (OLT). Investigate the relationship among minute ventilation $\left(\mathrm{V}_{\mathrm{E}}\right)$ variability, RPE, and walking endurance.

Setting Laboratory.

Methods A 6-min CWR was used as a warm-up preceding a CWR, at the same walking speed, until volitional fatigue or 30 min. Breath-by-breath ventilatory data were examined during the second CWR using a mono-exponential model. $V_{\mathrm{E}}$ variability was calculated as the difference between the observed and predicted values. Data were time-matched before and after 12 and 24 weeks of OLT. A Pearson's correlation was used for $V_{E}$ variability, RPE, and walking endurance.

Results A warm-up CWR did elicit a phasic ventilatory response. OLT resulted in faster ventilatory kinetics. Ventilatory variability reduced after 12 weeks of OLT but returned to pre-OLT values after 24 weeks of training. The change in $\mathrm{V}_{\mathrm{E}}$ variability was correlated with the change in RPE throughout the study. 12 and 24 weeks of OLT resulted in significant improvements in treadmill walking time.

Conclusions SCI patients can achieve a phasic ventilatory response to walking if the exercise bout is preceded by a warmup. OLT normalizes the ventilatory kinetics and improves walking endurance. The change in $\mathrm{V}_{\mathrm{E}}$ variability is correlated to RPE.
\end{abstract}

Supplementary information The online version of this article (https:// doi.org/10.1038/s41394-020-00346-6) contains supplementary material, which is available to authorized users.

Gino S. Panza

gpanza@med.wayne.edu

1 John D. Dingell Veterans Affairs Medical Center, Detroit, MI, USA

2 Department of Physiology, Wayne State University School of Medicine, Detroit, MI, USA

3 Department of Rehabilitation Science, George Mason University, Fairfax, VA, USA

\section{Introduction}

Individuals with a spinal cord injury (SCI) suffer from multi-system dysfunction stemming from altered afferent and efferent neural communication. Functional decline following SCI is well documented, specifically the loss of walking economy [1], walking speed [2], and endurance [3]. The reduced ability to ambulate and subsequent deconditioning lead to early mortality primarily through respiratory complications; although better medical care has increased longevity for these individuals, the gain in decades has been accompanied by an increased risk of ageassociated cardiovascular conditions and other age-related morbidity $[4,5]$.

Physiological data during volitional unaided walking in $\mathrm{SCI}$ is scant. It is becoming increasingly evident overground locomotor training can improve many physiological 
processes and may be superior if conducted under full weight-bearing compared to body weight supported training [6]. In patients capable of walking, locomotor training has shown to improve pulmonary function as evidenced by spirometric testing [7] and improved muscle activation [8]. However, despite the improvements in pulmonary function following locomotor training, it remains unknown if the pulmonary system limits treadmill walking endurance in patients with motor incomplete SCI (iSCI); more specifically during walking with no body weight support.

In healthy individuals, the ventilatory response to walking is curvilinear and can be deconstructed into 3 phases. In Phase I, there is an immediate rise in ventilation from baseline. Phase 2 exhibits a curvilinear rise which, if under the anaerobic threshold, evolves into a linear steady-state response (Phase 3). Previous exercise (e.g., warm-ups) and higher exercise intensities result in a faster ventilatory response (measured as $\tan [\tau]$ ) as the amplitude of the response is affected by ventilated oxygen consumption $\left(\mathrm{VO}_{2}\right)$ [9, 10]. In patients with cervical incomplete SCI (iSCI), our lab has previously reported improved overground walking speed, decreased ventilated oxygen consumption $\left(\mathrm{VO}_{2}\right)$, and ventilated carbon dioxide production $\left(\mathrm{VCO}_{2}\right)$ during treadmill walking following full weightbearing overground locomotor training (OLT) [11]. More specifically, during volitional treadmill walking at preferred walking speed, and without a warm-up, we observed a linear pulmonary minute ventilation $\left(\mathrm{V}_{\mathrm{E}}\right)$ response to constant work rate (CWR) [12]. In addition, we found a high degree of ventilatory variability that was reduced following 12 weeks of OLT without a change in average ventilation [12]. Additional pilot work suggested participants with cervical iSCI could achieve a phasic response if exercise was preceded by a warm-up walking bout in conjunction with an increase in exercise intensity (e.g., walking speed) [13]. These data indicate that despite neurological damage of the spinal cord at or above where the phrenic nerve exits the spinal column, individuals with cervical motor incomplete injuries can achieve a phasic response. Under these conditions, there was also a reduction in ventilatory variability, end-tidal $\mathrm{CO}_{2}$, and $\mathrm{VCO}_{2}$. The decreased $\mathrm{V}_{\mathrm{E}}$ variability was accompanied by improved ventilatory kinetics (faster), reduction in perceived exertion (RPE), and work of breathing $(\mathrm{Wb})$. Interestingly, the $\tau$ of $\mathrm{V}_{\mathrm{E}}$, and ventilatory variability correlated with RPE which may suggest the ventilatory response to exercise in these patients may impact perceived exertion and subsequently walking endurance when participants walk at above preferred walking speed. However, it remains unknown if the more intense walking speed or the warm-up bout of exercise elicited the hyperneic ventilatory response. Moreover, it remains unknown if the ventilatory variability indeed impacts treadmill walking endurance. We sought to describe these relationships and their adaptation during continued OLT.

\section{Methods}

\section{Study design and participants}

This intervention for this study was designed to elicit mechanical and metabolic adaptations for participants with iSCI with the aim of improving independent mobility. Specific to our aims regarding a better understanding of the ventilatory response to exercise, a secondary analysis was conducted to investigate the repeated effects of OLT on the ventilatory response to unaided treadmill walking at preferred walking speed. A pre- vs post 1 vs post 2-test design was used. Participants completed two 12-week cycles of OLT that was developed. Inclusion criteria was: $18+$ years or older, AIS C or D, must have a chronic injury operationalized as 1 or more years after injury, and participants had to be able to stand and initiate a step with or without an assistive ambulation aid. However, this assistive device could not include electrical stimulation. Participants were excluded if they had known cardiovascular, pulmonary, or metabolic disease as well as severe concomitant secondary complications following their injury (e.g. spasms/contractures).

\section{Overground locomotion training}

Details of the OLT program have been published elsewhere $[11,14]$. In short, the training focused on part-to-wholepractice sequences developed on the task-specific actions of the gait cycle. Sessions were $90 \mathrm{~min}$, two times a week, for 12 weeks. Each session had five primary training segments: joint mobility; volitional muscle activation; task-isolation; task-integration; and activity rehearsal. Participants were weight bearing at all times but did utilize the help from trainers when needed. No electrical stimulation was used. All sessions were conducted by two trained personnel.

\section{Treadmill testing protocol}

The treadmill test was conducted before and after the 12 weeks of OLT (described above). Participants completed a 2-bout square wave protocol for this study. Initially, participants stood for 3-5 min prior to a 6-min walking bout at a self-selected pace. The instructions for this pace were to select a speed which the participants felt mimicked their ambulatory speed off the treadmill, and that they could maintain safely. This walking bout was used as a warm-up. After the 6-min bout participants had 6 min of recovery. Participants were offered a seat during this time if they preferred. If they did sit, they were required to stand for the 
2-min preceding the next bout. The second, and final, working bout was at the same self-selected pace. However, participants were instructed to walk until volitional fatigue, or $30 \mathrm{~min}$, whichever came first. Thirty minutes was chosen as we hypothesized no participants would be able to walk for $30 \mathrm{~min}$. Participants were allowed to use handrails for balance but were instructed to minimize the amount of bodyweight they supported with their arms. Ratings of perceived exertion, on a $0-10$ scale [15], were obtained every 2 min during the walking bout.

\section{Instruments}

Participants' height and weight were measured with a stadiometer (SECA 213) and scale (Health O Meter 400KL). Breath-by-breath cardioventilatory parameters were collected with an Ultima CardiO2 gas exchange system (MedGraphics, Saint Paul, MN). These variables included, but were not limited to, ventilated oxygen and carbon dioxide $\left(\mathrm{VO}_{2}, \mathrm{VCO}_{2}\right.$, respectively), $\mathrm{V}_{\mathrm{E}}$, Tidal Volume $\left(\mathrm{V}_{\mathrm{T}}\right)$ and breathing frequency $\left(\mathrm{B}_{\mathrm{f}}\right)$. A 12-lead electrocardiogram (ECG; Mortara M12A, Milwaukee, WI) was monitored throughout exercise for safety purposes. Flow calibration was completed using a $3000 \mathrm{~mL}$ calibration syringe and gas calibrations were performed through the system's gas auto calibration configuration.

\section{Data analysis}

Data from the warm-up bout is not included as the warm-up bout was used as a perturbation to potentially elicit a phasic ventilatory response. Therefore, the analysis only included data from the second bout of CWR to volitional fatigue. All data processing was performed using the graphing software Origins (Origin 2016, OriginLab Corporation, Northampton, MA) on raw (unaveraged) $\mathrm{V}_{\mathrm{E}}$. Data were fit to the entire walking bout using a kinetic curve developed for moderate intensity exercise (Eq. 1). In the equation, $\mathrm{Y}(\mathrm{t})$ represents $\mathrm{V}_{\mathrm{E}}$ as a function of time, $\mathrm{Y}$ (baseline) is the average ventilation for $180 \mathrm{~s}$ prior to exercise, amplitude is the difference between resting data and the end-exercise data, time delay (TD) reflects the elapsed time until the exponential fit began, and the $\tau$ is the time taken to reach $63 \%$ of the archived amplitude.

$$
Y(t)=\text { YBaseline }+ \text { Amplitude }\left(1-e^{-(t-T D p) / \tau p}\right)
$$

In addition, prediction bands of $99 \%$ were fit to the data. Any data points outside of these bands were removed from the data and the curve was recalculated. These data were removed as they were assumed to be aberrant breaths from sighs, coughs, or talking. However, participants were instructed to refrain from talking unless required. Data were considered phasic of the curve converged to the data. Ventilatory variability were computed from taking the absolute value of the difference between observed data and the predicted data (Eq. 2). To maintain similar time-periods as RPE, ventilatory variability was averaged every $2 \mathrm{~min}$.

$$
\mid \text { Variability } \mid=\left(\operatorname{Observed}\left(\mathrm{V}_{\mathrm{E}}\right)-\operatorname{Predicted}\left(\mathrm{V}_{\mathrm{E}}\right)\right)
$$

The estimated work of breathing $(\mathrm{Wb})$ was calculated for each breathing using Eq. (3) [16], which has been used in patients with SCI [17]. The $\mathrm{Wb}$ was also averaged over the entire bout, and every $2 \mathrm{~min}$ increment.

$$
\text { Estimated } \mathrm{Wb}=-0.430+0.0504\left(\mathrm{~V}_{\mathrm{E}}\right)+0.00161\left(\mathrm{~V}_{\mathrm{E}}\right)^{2}
$$

\section{Statistical analysis}

Effect sizes were used to quantify the changes in all parameters following OLT. Cohen's d (effect size $=d$ ) was chosen. A $d$ of $0.2,0.5$ and 0.8 represent a small, medium and large effect size, respectively [18]. A group of age, height, and weight-matched able-bodied participants was used as a reference group for this study. A two-sided students t-test was used to determine if there were differences in height, weight, age, or body mass index (BMI) between the iSCI group and healthy reference group. No other comparisons between the two groups were made. The ablebodied group was included for reference purposes only. Outcome variables were only compared during matched walking speed bouts. All data were averaged over the entire exercise bout as well as time-matched for the shortest bout. The time-matched data is presented in the primary manuscript as the entire bout averages are presented in the Supplementary file. Correlations were deemed significant based on an alpha of 0.05 from Pearson's correlations. Data are presented as mean (SD). However, for graphing purposes the mean and standard error are presented.

\section{Results}

There were ten participants in the OLT program who had been tested at baseline, after 12 weeks of training, and then after 24 weeks of training using this study's particular testing protocol. However, two of these participants had sustained lumbar injuries. Due to the neuroanatomical organization of the ventilatory musculature, these participants were removed from the present analysis. Therefore, the data of only eight participants were appropriate to be included in these particular analyses. Participant characteristics can be found in Table 1. No changes in weight were recorded after OLT. There were no significant differences 
Table 1 Participant characteristics.

\begin{tabular}{llllllll}
\hline & Age (years) & Sex (M/F) & $\begin{array}{l}\text { Height } \\
\text { (meters) }\end{array}$ & $\begin{array}{l}\text { Weight } \\
\text { (kilograms) }\end{array}$ & BMI & $\begin{array}{l}\text { Time Since Injury } \\
\text { (months) }\end{array}$ & $\begin{array}{l}\text { Injury Level (number } \\
\text { C/D) }\end{array}$ \\
\hline iSCI & $45(16.3)$ & $8 / 0$ & $1.8(0.1)$ & $80.1(17.3)$ & $24.3(4.0)$ & $44.3(17.3)$ & AIS \\
AB & $34.6(11.3)$ & $7 / 1$ & $1.8(0.06)$ & $81.8(13.95)$ & $26.0(4.2)-$ & - & - \\
\hline
\end{tabular}

Data are presented as mean (SD).

$B M I$ body mass index $\mathrm{kg} / \mathrm{m}^{2}$, AIS American Spinal Injury Association Impairment Scale.

Table 2 Resting cardiopulmonary averages.

\begin{tabular}{|c|c|c|c|c|c|c|c|c|}
\hline & $\mathrm{AB}$ & & Pre & & Post 1 & & Post 2 & \\
\hline HR & 83.75 & (10) & 89.14 & (18) & 91.93 & $(9.4)^{*}$ & 85.88 & $(15)^{*, \# \#}$ \\
\hline $\mathrm{VO}_{2}(\mathrm{Abs})$ & 354.78 & (105) & 343.38 & (66) & 367.95 & $(76)^{*}$ & 341.32 & $(65)^{*, \#}$ \\
\hline $\mathrm{VO}_{2}(\mathrm{Rel})$ & 4.31 & $(0.80)$ & 4.41 & (1.0) & 4.66 & $(1.2)^{*}$ & 4.30 & $(1.00)^{*, \#}$ \\
\hline $\mathrm{VCO}_{2}(\mathrm{Abs})$ & 332.98 & (99) & 336.37 & (82) & 359.16 & $(83)^{*}$ & 360.83 & $(83)^{*}$ \\
\hline$V_{E}$ & 10.44 & (2.4) & 11.18 & (2.9) & 11.48 & $(2.45)^{*}$ & 11.95 & $(2.40)$ \\
\hline $\mathrm{V}_{\mathrm{T}}$ & 828.79 & $(218)$ & 652.82 & (132) & 675.87 & $(91)^{*}$ & 683.16 & $(145)^{*}$ \\
\hline $\mathrm{B}_{\mathrm{f}}$ & 13.32 & (2.7) & 17.95 & (3.6) & 16.65 & $(2.40)^{*}$ & 19.26 & 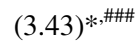 \\
\hline $\mathrm{W}_{\mathrm{B}}$ & 0.38 & $(0.25)$ & 0.67 & $(0.76)$ & 0.45 & $(0.22)^{*}$ & 0.46 & $(0.27)^{*}$ \\
\hline $\mathrm{V}_{\mathrm{E}}$ Var & 2.92 & (1.4) & 2.60 & $(0.65)$ & 3.03 & $(1.2)^{*}$ & 2.97 & $(1.44)^{*}$ \\
\hline
\end{tabular}

Resting cardiopulmonary data prior to CWR walking to volitional fatigue. $*, * *, * * *=$ small, medium, and

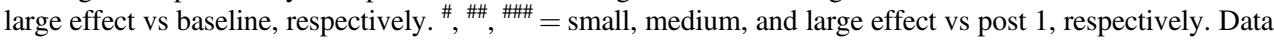
are presented as mean $(\mathrm{SD})$.

between the two groups. Two participants did increase their walking speed after the first 12 weeks of OLT.

Resting cardiopulmonary variables can be found in Table 2 . Small effects were found for all the resting cardiopulmonary variables in Table 2 after 12 weeks of OLT. A small increase in resting heart rate, $\mathrm{VO}_{2}, \mathrm{VCO}_{2}, \mathrm{~V}_{\mathrm{E}}, \mathrm{V}_{\mathrm{T}}, \mathrm{V}_{\mathrm{E}}$ variability was found as a small reduction in $\mathrm{B}_{\mathrm{f}}$ and the work of breathing were found. An additional 12 weeks of OLT, when compared to baseline, resulted in a small decrease in heart rate, $\mathrm{VO}_{2}$, and work of breathing, with a small increase in $\mathrm{VCO}_{2}, \mathrm{~V}_{\mathrm{E}}, \mathrm{V}_{\mathrm{T}}, \mathrm{B}_{\mathrm{f}}$, and $\mathrm{V}_{\mathrm{E}}$ variability. However, when compared to the first 12-week cycle of OLT, a small reduction in heart rate and $\mathrm{VO}_{2}$ was found as well as a large increase in resting $\mathrm{B}_{\mathrm{f}}$.

\section{Ventilatory kinetics}

Ventilatory kinetics data are presented in Fig. 1. A small effect on resting ventilation was found following 12 and 24 weeks of OLT. A medium effect on the speed of the amplitude of ventilation was found after 12 weeks of OLT. However, after an additional 12 weeks of OLT training, the amplitude returned to baseline values. The change from Post 1 to Post 2 did result in a large effect. The tau of minute ventilation did reduce (large effect) following 12 weeks of OLT. After an additional 12 weeks of training the tau remained lower than pre-OLT values (large effect). However, there was a rise (medium effect) from Post 1 to
Post 2 following an additional 12 weeks of OLT. Large effects were also found when standardizing the tau to the amplitude and vice versa (Fig. 1c).

Secondary averaged time-matched cardiopulmonary variables can be found in Table 3. There was a small reduction in $\mathrm{VO}_{2}$ and $\mathrm{V}_{\mathrm{T}}$ found after 12 weeks of OLT, but no other differences were found. An additional 12 weeks of OLT resulted in a small reduction in heart rate when compared to baseline and Post 1 measures. A small increase in $\mathrm{V}_{\mathrm{E}}, \mathrm{B}_{\mathrm{f}}$, and the work of breathing was found when compared to baseline measures. When comparing Post 2 to Post 1 , there was a small increase in $\mathrm{VO}_{2}, \mathrm{VCO}_{2}, \mathrm{~V}_{\mathrm{T}}$, work of breathing and a medium increase in $\mathrm{B}_{\mathrm{f}}$.

Following 12 and 24 weeks of OLT, there was a medium and large, respectively, reduction in RPE. There was also an additional small reduction in RPE from Post 1 to Post 2. A large reduction in ventilatory variability was found after 12 weeks of OLT. However, the ventilatory variability returned to baseline values after an additional 12 weeks of OLT. A small effect was found on walking endurance after 12 weeks of OLT. A medium effect on walking endurance was also found when compared to baseline measures during the Post 2 test, and when Post 2 test was compared to Post 1.

Figure 2 shows the positive correlations between $\mathrm{V}_{\mathrm{E}}$ variability, $\mathrm{VCO}_{2}, \mathrm{VO}_{2}$ and the relationship between the change in $\mathrm{V}_{\mathrm{E}}$ variability and the change in RPE for the entire study. After 12 weeks of OLT the change in tau did 
Fig. 1 Difference in the ventilatory kinetics following 12 and 24 weeks of OLT in SCI patients with reference values from matched ablebodied control participants. Differences in baseline and amplitude of $\mathrm{V}_{\mathrm{E}}$ is presented in a. Differences in the speed of ventilatory kinetics is presented is b. Differences in the ventilatory kinetics when tau is normalized to amplitude and when amplitude is normalized to tau is presented in c. $* * *, * * *=$ small, medium and large effect compared to baseline values. \#, \#\#, \#\#\# = small, medium, and large effect when Post 2 data is compared to Post 1 data.
Table 3 Time-Matched and averaged cardiopulmonary variables.
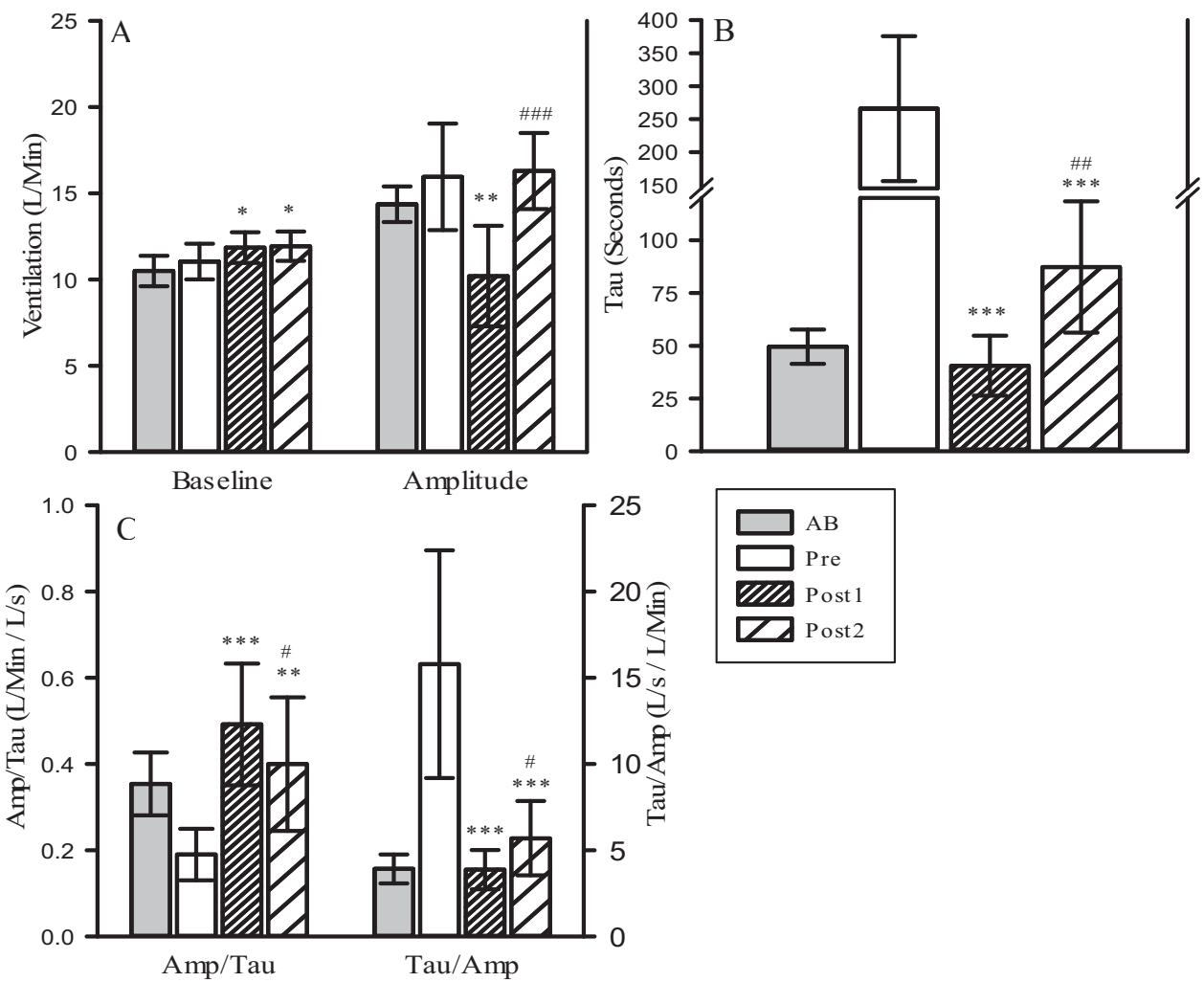

\begin{tabular}{lrlrlrlrl}
\hline & \multicolumn{1}{c}{ AB } & \multicolumn{3}{c}{ PRE } & & Post 1 & \multicolumn{3}{c}{ Post 2 } \\
\hline $\mathrm{HR}$ & 102.45 & $(13)$ & 111.09 & $(21)$ & 110.94 & $(15)$ & 105.60 & $(17)^{* \#}$ \\
$\mathrm{VO}_{2}$ (Abs) & 1066.39 & $(260)$ & 945.60 & $(417)$ & 809.56 & $(409)^{*}$ & 930.40 & $(354)^{\#}$ \\
$\mathrm{VO}_{2}$ (Rel) & 10.03 & $(2.1)$ & 11.98 & $(4.84)$ & 11.06 & $(4.56)^{*}$ & 11.39 & $(3.7)$ \\
$\mathrm{VCO}_{2}$ (Abs) & 937.85 & $(233)$ & 830.44 & $(339)$ & 785.57 & $(353)$ & 851.87 & $(319)^{\#}$ \\
$\mathrm{~V}_{\mathrm{E}}$ & 24.19 & $(4.53)$ & 23.44 & $(6.31)$ & 22.57 & $(9.2)$ & 25.81 & $(8.7)^{* \#}$ \\
$\mathrm{~V}_{\mathrm{T}}$ & 1257.37 & $(302)$ & 1016.39 & $(298)$ & 934.51 & $(234)^{*}$ & 1002.58 & $(331)^{\#}$ \\
$\mathrm{~B}_{\mathrm{f}}$ & 19.95 & $(4.13)$ & 25.61 & $(7.0)$ & 24.95 & $(4.8)$ & 27.34 & $(6.3)^{* \# \#}$ \\
$\mathrm{~W}_{\mathrm{B}}$ & 1.79 & $(0.61)$ & 1.78 & $(0.90)$ & 1.70 & $(1.4)$ & 2.10 & $(1.3)^{* \#}$ \\
\hline
\end{tabular}

Averaged time-matched cardiopulmonary variables during the CWR to volitional fatigue.

Data are presented as mean (SD).

$*, * *, * * *=$ small, medium, and large effect vs baseline, respectively.

${ }^{\#,}{ }^{\# \#, ~}{ }^{\# \#}=$ small, medium, and large effect vs post 1 , respectively. not correlate to walking endurance in those that walked to volitional fatigue (Coefficient $=0.28, R^{2}=0.08, P=0.65$ ). However, after 24 weeks of OLT the change in tau from Post 2 to Post 1 correlated with the change in walking endurance (Coefficient $=0.89, R^{2}=0.79, P=0.04$ ). More specifically, in the five individuals that walked to volitional fatigue after 12 weeks of OLT, the change in $\mathrm{V}_{\mathrm{E}}$ variability correlated with the change in walking endurance (Coefficient $\left.=-0.97, R^{2}=0.95, P=0.005\right)$. After an additional 12 weeks of training, 6 individuals walked to volitional fatigue (see Methodological Considerations below), but no relationship was found between ventilatory variability and walking endurance $\left(\right.$ Coefficient $=0.61, R^{2}=0.38, P=0.19$ ). Due to the change between 12 and 24 weeks of OLT, there was no relationship found between the change in $V_{E}$ variability and the change in walking endurance (Coefficient $=0.25, R^{2}=0.06, P=0.46$ ).

\section{Discussion}

The results of this study show that when unsupported treadmill walking is preceded by a 6-min warm-up bout of treadmill walking, but without a change in exercise intensity 

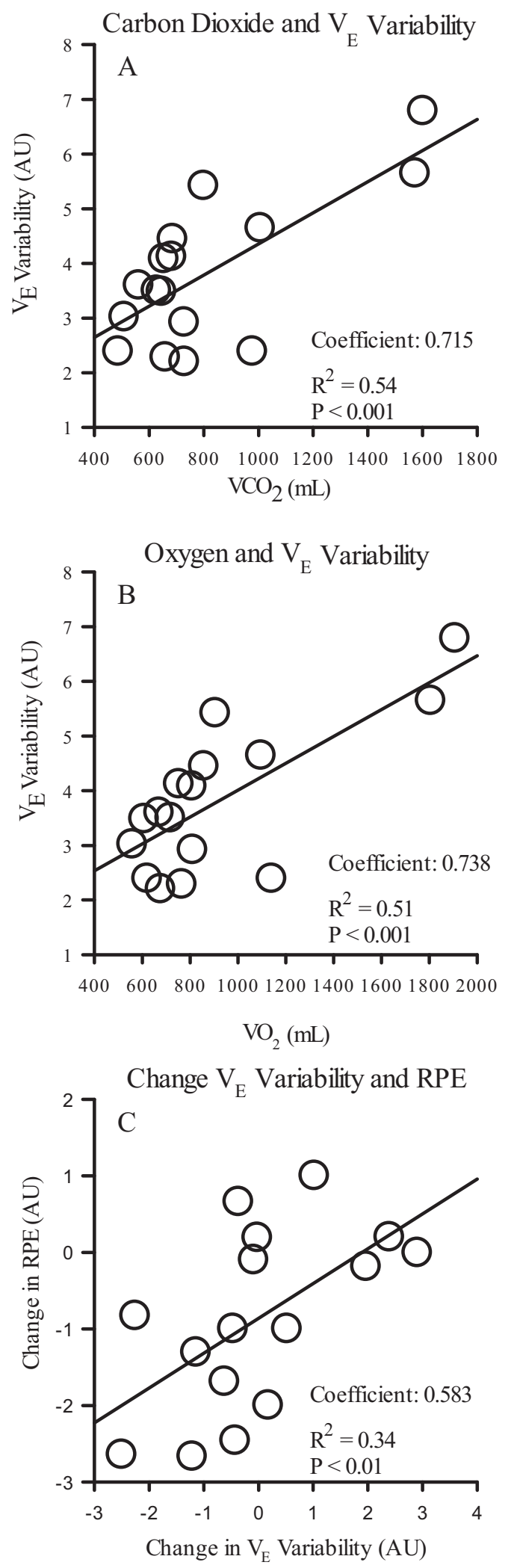

Fig. 2 Correlations between the changes in $\mathrm{VO}_{2}, \mathrm{VCO}_{2}$ and $\mathrm{V}_{\mathrm{E}}$ variability between Post 1-Pre and Post 2-Post 1 are presented in $\mathbf{a}$ and $\mathbf{b}$. The correlation between the change in RPE and $V_{E}$ variability can be found in c. Pearson's correlations were used with significance set at an alpha of 0.05 . (i.e., speed), participants with iSCI can achieve a phasic response. In this case, a phasic response was measured by the fact that Eq. (1) fit the data thus producing the measures typically reported in kinetics. In addition, the ventilatory response to exercise is accelerated after 12 and 24 weeks of OLT, with concomitant improvements in walking endurance and reductions in RPE after 12 and 24 weeks of OLT. Ventilatory variability reduced at 12 weeks, but returned to pre-OLT values after an additional 12 weeks of training despite the continued reduction in RPE and improvements in walking endurance. Finally, our data show that the $V_{E}$ variability is correlated with overall metabolic activity during exercise and that the changes in $\mathrm{V}_{\mathrm{E}}$ variability are correlated with the changes in RPE (see Fig. 2).

Studies investigating the ventilatory kinetics and its response to training modalities during unaided walking in participants with motor iSCI are scant. Currently, we are unaware of any studies investigating these responses in patients capable of treadmill walking without body weight support. Previously, we had reported an atypical ventilatory response (linear) to preferred walking speed in participants with cervical motor incomplete SCI, but found a reduction in ventilatory variability following 12 weeks of OLT [11]. In a subsequent pilot study, we investigated if a warm-up bout of exercise coupled with increased walking speed (e.g., intensity) could elicit a phasic response to exercise, and reported participants with iSCI could achieve a phasic response and that 12 weeks of OLT resulted in a faster ventilatory response with concurrent reduction in ventilatory variability and RPE [12]. In addition, we reported significant relationships between ventilatory variability and RPE and suggested that ventilatory variability may have an impact on treadmill walking endurance at above preferred walking speeds [12]. We now report that a phasic response can be achieved with the same exercise intensity if preceded by a warm-up bout of walking, and that 12 and 24 weeks of OLT accelerates the ventilatory response to exercise, reduces RPE, and reduces ventilatory variability at 12 , but not 24 weeks, at a lower exercise intensity. It remains unknown why ventilatory variability returned to pre-OLT levels following the second 12 weeks of OLT. The likely explanation is that the treadmill walking speed is no longer in the same exercise intensity domain as the pre-OLT and post 1 testing timepoints, and the reduction in exercise intensity allows for a more robust ventilatory response to exercise without further impact on other systems (e.g., sympathetic activation). In other words, the variability in minute ventilation has less impact on the overall physiological response to treadmill walking. In addition, we confirm that there is a relationship between ventilatory variability and RPE. However, this relationship is weaker than in our previous report. A possible explanation for the weaker relationship is the lower walking intensity and changes in the exercise intensity domain. 
Our current report indicates that ventilatory variability may have a significant impact on walking endurance, and may be linked to the overall metabolic work performed during walking in these participants.

Functional electrical stimulation (FES) has been shown to elicit a phasic ventilatory response and changes following FES training. In six patients with complete injuries (four above $\mathrm{T} 7$, one at $\mathrm{T} 5$, and one at $\mathrm{T} 4$ ), using functional electrical simulation, Barstow reported the mean response time for ventilatory on and off kinetics and reported significantly slowed ventilatory kinetic responses $(202 \pm 61 \mathrm{~s})$ [19] which are comparable to our pre-OLT values of $265.96 \pm 310.07$ (mean \pm standard deviation), but with significantly lower variability. Thereafter, during unloaded cycling with FES Barstow reported a mean response time of $186 \mathrm{~s}$ which reduced to $117.3 \mathrm{~s}$ after 24 exercise sessions (3 per week) that lasted $30 \mathrm{~min}$. However, after training when relative workload was controlled for, a non-significant decrease in the ventilatory mean response time was reported (166.8 \pm 38.9$)$ [20]. These earlier papers show that participants with an SCI can achieve a phasic ventilatory response when FES is used to facilitate the limb movements, and that the phasic response is malleable. Our results suggest that these relationships are also present in individuals capable of walking, and with injuries levels that could affect the nerves innervating the muscles of breathing.

The correlations between the changes in $V_{E}$ variability and RPE indicate that the variability of $\mathrm{V}_{\mathrm{E}}$ may be an important factor in determining RPE and treadmill walking endurance in these participants. Thus, understanding the variables that impact ventilatory variability could provide useful therapeutic targets during the rehabilitation effort for these participants. Unfortunately, direct evidence of different physiological variables and their effects on ventilatory variability during exercise remains unknown. We previously suggested that locomotor respiratory coupling (LRC) [21], ventilatory muscle fatigue, and the respiratory steal phenomenon [22-27] may impact ventilatory variability and treadmill walking endurance in patients with iSCI. In addition, dynamic hyperinflation may impact both ventilatory variability and treadmill walking endurance as Gee et al. reported improvements in pulmonary function and exercise capacity with reduced dynamic hyperinflation during arm-crank exercise following ventilatory muscle training [28]. However, as we do not have direct evidence supporting any of these attributions, these potential correlations or inferences about causality are admittedly speculative and serve as potential hypotheses for future research.

Despite the fact that respiratory function after spinal cord injury is highly studied, the ventilatory kinetics during exercise remains understudied. Therefore, it is difficult to provide direct clinical benefits of improved ventilatory kinetics. However, the improved ventilatory kinetics were concurrent with improved exercise capacity as well as a reduction in the work of breathing and RPE (see Fig. 3 and Table 2). In addition, locomotor training has been shown to improve pulmonary function $[7,29]$ in spinal cord injured patients. Furthermore, respiratory muscle training has been shown to improve ventilatory function and reduce dynamic hyperinflation during exercise [28] in participants with spinal cord injuries. Finally, in trained Paralympic athletes, inspiratory muscle training resulted in improvements in maximum voluntary ventilation and maximum exercise capacity [30]. Despite no changes in RPE or dyspnea, dyspnea was always lower than RPE [30]. Therefore, it may be possible that the improvements in ventilatory function,
Fig. 3 Differences in timematched RPE, $V_{E}$ variability and walking endurance. RPE and $V_{E}$ variability are presented on the left $\mathrm{Y}$-axis and walking endurance is presented in seconds on the right $\mathrm{Y}$-axis. $*, * *, * * *=$ small, medium and large effect compared to baseline values. " , \#\#, \#\#\#= small, medium, and large effect when Post 2 data is compared to Post 1 data.

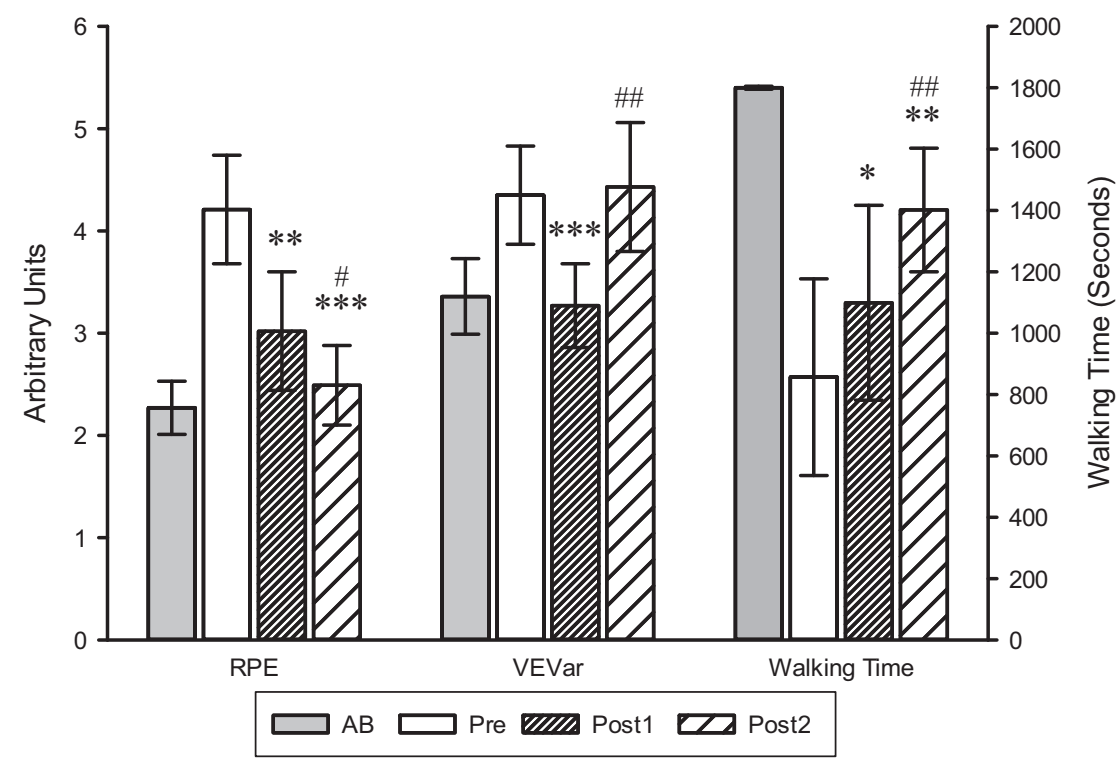


reduced work of breathing, and reduced RPE in these participants may also impact dyspnea. However, this remains to be studied when examining ventilatory kinetics.

\section{Methodological considerations}

There are several methodological issues that must be considered as limitations on the interpretation of our findings. With respect to the fit of the kinetic equation, the TD is typically left unchanged during the computation of the kinetic curve. In our study we chose to restrict TD to 0 if it did not naturally fall between -5 and $5 \mathrm{~s}$ (see Supplementary Fig. 2). Our reasoning was twofold. First, TD is used to account for the time required for muscle oxidative changes to be measured in ventilated air. However, this is not the case when measuring minute ventilation as immediate increases in ventilation are seen during exercise and even during passive limb movement [31-33]. With an uncontrolled TD two of our iSCI participants' data produced obviously incorrect data. For example, one participant had a tau of over 1 million. In addition, at the end of the second 12 weeks of OLT the kinetics curve would not converge (i.e., fit) 5 of the 8 participants' data despite a visually phasic ventilatory response. However, in all cases, if the TD was set to 0, all curves fit. Our initial approach was to restrict TD based on the current literature on the control of ventilation during exercise [32]. However, the data was re-analyzed with an unfixed TD to ensure appropriate analysis by visual inspection.

Another curve fitting limitation must also be addressed. Typically, in pulmonary $\mathrm{VO}_{2}$ kinetics, curves are fit to 3 or 6 min of data. In the current report, the curve was fit to the entire data set. Supplementary Figure 1 provides a visual representation on why we elected to fit the curve to the entire exercise bout. In addition, in two SCI participants, pre-OLT did have an obvious deflection in their $\mathrm{V}_{\mathrm{E}}$ indicating they surpassed their anaerobic threshold. In these two cases, the curve was fit to the deflection point, and a linear regression was applied for the remainder of the data, and variability was computed the same as the kinetic equation. Lastly, in the five participants who walked to volitional fatigue, the change in ventilatory variability correlated with walking endurance. The participants who were removed from this correlation were as follows: two participants removed from the correlation were stopped early during their post-test treadmill walk due to deteriorating mechanics during walking to which the research assistant ended the CWR bout. The other participant walked the full $30 \mathrm{~min}$ pre and post, thus did not walk to volitional fatigue. This was an unexpected result as we did not expect individuals to be able to walk $30 \mathrm{~min}$ without bodyweight support. The latter point addresses the final methodological concern. Changes in exercise intensity are known to affect ventilatory kinetics, and it remains unknown if the variability in $\mathrm{V}_{\mathrm{E}}$ is different at different exercise intensities. In addition, the changes in exercise intensity will impact walking endurance, and thus the data points collected during exercise bouts for participants that finished the full 30-min (Post $1=3$, Post $2=4$ ), may not fully represent the impact of systemic fatigue on ventilatory variability and RPE, thus confounding the correlations. However, a reduction in exercise intensity (i.e., intensity domain) is a positive result, but it may impact the correlations presented here. Therefore, future studies investigating similar variables should not restrict the duration of the CWR bout.

In conclusion, our data show that participants with a motor incomplete spinal cord injury about $\mathrm{T} 7$ achieved a phasic ventilatory response to unaided treadmill walking at lower intensities when the walking bout was preceded by a 6-min warm-up bout. In addition, 12 and 24 weeks of OLT led to improvements in ventilatory kinetics, RPE, and walking endurance. Furthermore, ventilatory variability reduced after 12 weeks of OLT, but returned to preOLT values after an additional 12 weeks of OLT, and that the ventilatory variability is related to both $\mathrm{VO}_{2}$ and $\mathrm{VCO}_{2}$. Lastly, we confirmed that the changes in ventilatory variability are associated with changes in RPE and walking endurance, and thus ventilatory variability may provide an avenue for new therapeutic approaches for participants with motor iSCI.

\section{Data availability}

Data sharing is not applicable to this article as no datasets were generated or analyzed during the current study.

Funding There was no funding for this work. However, GSP is currently supported by Department of Veteran Affairs (IK1RX00294502).

Author contributions GSP and AAG helped develop the overground locomotor training program and primary research questions. GSP administered training and conducted the cardiopulmonary exercise tests. GSP analyzed the data. GSP and AAG constructed and reviewed the manuscript. GSP and AAG approved the final version of the manuscript.

Ethical approval All study procedures were approved by the Institutional Review Board at George Mason University (618911). The study conformed to the standards set by the Declaration of Helsinki, except for registration in a public database. All participants provided written consent prior to enrollment in the study.

\section{Compliance with ethical standards}

Conflict of interest The authors declare that they have no conflict of interest.

Publisher's note Springer Nature remains neutral with regard to jurisdictional claims in published maps and institutional affiliations. 


\section{References}

1. Waters RL, Yakura JS, Adkins RH. Gait performance after spinal cord injury. Clin Orthop. 1993;288:87-96.

2. Field-Fote EC, Roach KE. Influence of a locomotor training approach on walking speed and distance in people with chronic spinal cord injury: a randomized clinical trial. Phys Ther. 2011;91:48-60.

3. Bassett DR, Howley ET. Limiting factors for maximum oxygen uptake and determinants of endurance performance. Med Sci Sports Exerc. 2000;32:70-84.

4. 2015 SCI Fact Sheet. (2016). https://www.nscisc.uab.edu/ PublicDocuments/reports/pdf/2015\%20NSCISC\%20Annual\% 20Statistical\%20Report\%20Complete\%20Public\%20Version.pdf.

5. Garshick E, Kelley A, Cohen SA, Garrison A, Tun CG, Gagnon $\mathrm{D}$, et al. A prospective assessment of mortality in chronic spinal cord injury. Spinal Cord. 2005;43:408-16.

6. Hornby TG, Reisman DS, Ward IG, Scheets PL, Miller A, Haddad D, et al. Clinical practice guideline to improve locomotor function following chronic stroke. Incomplete Spinal Cord Inj, Brain Inj Pre. 2020;44:49-100.

7. Tiftik T, Gökkaya NKO, Malas FÜ, Tunç H, Yalçın S, Ekiz T, et al. Does locomotor training improve pulmonary function in patients with spinal cord injury? Spinal Cord. 2015;53:467-70.

8. Terson de Paleville D, McKay W, Aslan S, Folz R, Sayenko D, Ovechkin A. Locomotor step training with body weight support improves respiratory motor function in individuals with chronic spinal cord injury. Respir Physiol Neurobiol. 2013;189:491-7.

9. Casaburi R, Barstow TJ, Robinson T, Wasserman K. Influence of work rate on ventilatory and gas exchange kinetics. J Appl Physiol Bethesda Md 1985. 1989;67:547-55.

10. Ward SA, Whipp BJ. Kinetics of the ventilatory and metabolic responses to moderate-intensity exercise in humans following prior exercise-induced metabolic acidaemia. Adv Exp Med Biol. 2010;669:323-6.

11. Gollie JM, Guccione AA, Panza GS, Jo PY, Herrick JE. Effects of overground locomotor training on walking performance in chronic cervical motor-incomplete spinal cord injury: a pilot study. Arch Phys Med Rehabil. 2017;98:1119-25.

12. Panza GS, Guccione AA, Chin LM, Gollie JM, Herrick JE, Collins JP. Effects of overground locomotor training on the ventilatory response to volitional treadmill walking in individuals with incomplete spinal cord injury: a pilot study. Spinal Cord Ser Cases. 2017;3:17011.

13. Panza GS, Herrick JE, Chin LM, Gollie JM, Collins JP, O'Connell DG, et al. Effect of overground locomotor training on ventilatory kinetics and rate of perceived exertion in persons with cervical motor-incomplete spinal cord injury. Spinal Cord Ser Cases. 2019;5:80.

14. Gollie JM, Guccione AA. Overground locomotor training in spinal cord injury: a performance-based framework. Top Spinal Cord Inj Rehabil. 2017;23:226-33.

15. Robertson RJ, Goss FL, Metz KF. Perception of physical exertion during dynamic exercise: a tribute to Professor Gunnar A. V. Borg. Percept Mot Skills. 1998;86:183-91.

16. Coast JR, Rasmussen SA, Krause KM, O'Kroy JA, Loy RA, Rhodes J. Ventilatory work and oxygen consumption during exercise and hyperventilation. J Appl Physiol Bethesda Md 1985. 1993;74:793-8.
17. Foll-de Moro DL, Tordi N, Lonsdorfer E, Lonsdorfer J. Ventilation efficiency and pulmonary function after a wheelchair intervaltraining program in subjects with recent spinal cord injury. Arch Phys Med Rehabil. 2005;86:1582-6.

18. Cohen J. Statistical power analysis for the behavioral science. 2nd ed. Routledge, New Your University, New York, New York. Lawrence Erlbau Associates, Publishers; 1988.

19. Barstow TJ, Scremin AM, Mutton DL, Kunkel CF, Cagle TG, Whipp BJ. Gas exchange kinetics during functional electrical stimulation in subjects with spinal cord injury. Med Sci Sports Exerc. 1995;27:1284-91.

20. Barstow TJ, Scremin AM, Mutton DL, Kunkel CF, Cagle TG, Whipp BJ. Changes in gas exchange kinetics with training in patients with spinal cord injury. Med Sci Sports Exerc. 1996;28: 1221-8.

21. Daley MA, Bramble DM, Carrier DR. Impact loading and locomotor-respiratory coordination significantly influence breathing dynamics in running humans. Plos ONE. 2013;8: e70752-70752.

22. Harms CA, Dempsey JA. Cardiovascular consequences of exercise hyperpnea. Exerc Sport Sci Rev. 1999;27:37-62.

23. Harms CA, Babcock MA, McClaran SR, Pegelow DF, Nickele GA, Nelson WB, et al. Respiratory muscle work compromises leg blood flow during maximal exercise. J Appl Physiol Bethesda Md 1985. 1997;82:1573-83.

24. Dominelli PB, Archiza B, Ramsook AH, Mitchell RA, Peters CM, Molgat-Seon Y, et al. Effects of respiratory muscle work on respiratory and locomotor blood flow during exercise. Exp Physiol. 2017;102:1535-47.

25. Mador MJ, Magalang UJ, Rodis A, Kufel TJ. Diaphragmatic fatigue after exercise in healthy human subjects. Am Rev Respir Dis. 1993;148(6 Pt 1):1571-5.

26. Johnson BD, Babcock MA, Suman OE, Dempsey JA. Exerciseinduced diaphragmatic fatigue in healthy humans. J Physiol. 1993;460:385-405.

27. Harms CA, Wetter TJ, McClaran SR, Pegelow DF, Nickele GA, Nelson WB, et al. Effects of respiratory muscle work on cardiac output and its distribution during maximal exercise. J Appl Physiol Bethesda Md 1985. 1998;85:609-18.

28. Gee CM, Williams AM, Sheel AW, Eves ND, West CR. Respiratory muscle training in athletes with cervical spinal cord injury: effects on cardiopulmonary function and exercise capacity. J Physiol. 2019;597:3673-85.

29. Sherman MFB, Lam T, Sheel AW. Locomotor-respiratory synchronization after body weight supported treadmill training in incomplete tetraplegia: a case report. Spinal Cord. 2009;47:896-8.

30. West CR, Taylor BJ, Campbell IG, Romer LM. Effects of inspiratory muscle training on exercise responses in Paralympic athletes with cervical spinal cord injury. Scand J Med Sci Sports. 2014;24:764-72.

31. Diamond LB, Casaburi R, Wasserman K, Whipp BJ. Kinetics of gas exchange and ventilation in transitions from rest or prior exercise. J Appl Physiol. 1977;43:704-8.

32. Forster HV, Haouzi P, Dempsey JA. Control of breathing during exercise. Compr Physiol. 2012;2:743-77.

33. Bell HJ, Duffin J. Respiratory response to passive limb movement is suppressed by a cognitive task. J Appl Physiol. 2004;97: 2112-20. 